\title{
ASSOCIAÇÃO ENTRE MEGAESOFAGO E CARCINOMA DE ESÔFAGO
}

\author{
Ademir Rocha, Hipólito de Oliveira Almeida, \\ Fued Elias Esper, Douglas Marra de Moraes, \\ Edivar Pereira dos Santos e Vicente de Paula Antunes Teixeira
}

\begin{abstract}
Analisa-se, em material de necropsias, a ocorrência de carcinoma esofágico em não-chagásicos e em chagásicos com e sem megaesôfago.

Observou-se um aumento altamente significativo da freqüencia do carcinoma de esôfago associado ao megaesôfago, em relação aos controles e aos chagásicos sem mẹgaesôfago. A associação entre carcinoma esofágico e infecção chagásica não foi estatisticamente significativa.
\end{abstract}

Palavras chaves: Megaesófago. Carcinoma de esôfago. Doença de Chagas, Câncer Necropsias.

O carcinoma de esôfago ( $\mathrm{CaE})$ é considerado, correntemente, como uma das complicações do megaesôfago (ME), seja o chagásico (MECh), seja o idiopático (MEI).

Ao revermos a literatura, constatamos a publicação de 54 casos da associação $\mathrm{CaE}-\mathrm{MECh}^{3}{ }^{5} 78$ 91612 . Em termos percentuais, as freqüências registradas de $\mathrm{CaE}$ no MECh variam de $1,1^{\prime}$ a $6,6 \%$ '. Não se tem avaliado, todavia, se tal associação é ocasional ou estatisticamente significativa. Uma outra possibilidade até agora não analisada é a de que o $\mathrm{CaE}$ se relacione à infecção chagásica, e não ao "mega"; em outras palavras, o indivíduo teria maior propensão ao $\mathrm{CaE}$ por ser chagásico, e não por ter ME.

Trabalhando em região endèmica da tripanossomose americana, na qual é freqüente o diagnós-

\footnotetext{
* Trabaiho dos serviços de Cirurgia e Anatomia Patológica do Centro de Ciências Biomédicas da Universidade Federal de Uberlândia, e da disciplina de Patologia Geral da Faculdade de Medicina do Triângulo Mineiro (Uberaba)

Endereço para correspondência: Ademir Rocha - Serviço de Antatomia Patológica do Centro de Ciências Biomédicas - Universidade Federal de Uberlândia - Campus Umuarama Uberlàndia - MG - 38.400

Recebido para publicação em 26-4-83
}

tico de ME, propomo-nos analisar, comparativamente, em material de necropsias, a ocorrência do $\mathrm{CaE}$ em não-chagásicos e em chagásicos com e sem $\mathrm{ME}$.

\section{MATERIAL E METODOS}

Foram revistos os protocolos de todas as necropsias efetuadas nos serviços de Anatomia Patológica do Centro de Ciências Biomédicas da Universidade Federal de Uberlândia e da Faculdade de Medicina do Triângulo Mineiro (Uberaba), no periodo de janeiro de 1956 a maio de 1982. Dada a raridade, na infância, tanto do $\mathrm{MECh}$, quanto do $\mathrm{CaE}$, apenas se consideraram as necropsias de indivíduos que tinham 15 ou mais anos de idade. Desprezaram-se os casos em que o tórax não foi examinado e em que a idade năo foi anotada. Com tais restrições, restou um total de 3.474 casos, que foram divididos nos seguintes grupos: chagásicos com $\mathrm{ME}$; chagásicos sem $\mathrm{ME}$; não-chagásicos. Cada um destes grupos foi analisado quanto à ocorrência ou não de $\mathrm{CaE}$, tanto globalmente quanto subdivididos por faixas etárias.

Dentre os carcinomas esofágicos, considerou-se apenas o carcinoma espinocelular (ou epidermóide, ou de células escamosas), já que os adenocarci- 
Rocha A, Almeida HO, Esper FE. Moraes DM, Santos EP, Teixeira VPA. A associaçào entre megaesôfago e carcinoma de esôfago. Revista da Sociedade Brasileira de Medicina Tropical 16:94-97, Abr-Jun, 1983

nomas verificados eram da região cárdica ou atingiam simultaneamente o esôfago e o estômago, de forma a não se poder excluir sua origem gástrica.

Nos estudos estatísticos, empregou-se o teste do $x^{2}$ (quiquadrado).

\section{RESULTADOS}

A avaliação dos 3.474 protocolos de necropsias selecionados e revistos mostrou os dados que constam das Tabelas 1 e 2 .
Segundo os dados da Tabela 1, a ocorrência de CaE foi significativamente maior no grupo de chagásicos com $\mathrm{ME}$ em relação aos demais. A subdivisào dos grupos por faixas etárias (Tabela 2) mostrou idêntico predomínio, exceto na década de 45 a 54 anos.

Tabela 1 - Ocorrência de carcinoma do esôfago (CaE) em necropsias de chagásicos com e sem megaesôfago ( $M E$ ), e não-chagásticos, no Triângulo Mineiro, no periodo de 1956-1982

\begin{tabular}{lcc}
\hline & $N^{o}$ de casos & Casos com CaE \\
\hline Chagásicos com ME & 107 & $5(4,6 \%)$ \\
Chagásicos sem ME & 1352 & $7(0,5 \%)$ \\
Não chagásicos & 2015 & $11(0,5 \%)$ \\
\hline
\end{tabular}

Tabela 2 - Ocorrência de carcinoma do esôfago (CaE) em necropsias de chagásicos com e sem megaesôfago, e nâochagásicos, por década etária, no Triângulo Mineiro, no periodo de 1956-1982

\begin{tabular}{|c|c|c|c|c|c|c|}
\hline \multirow{2}{*}{$\begin{array}{c}\begin{array}{c}\text { Grupo etário } \\
\text { (em anos) }\end{array} \\
15-24\end{array}$} & \multicolumn{2}{|c|}{$\begin{array}{c}\text { Chagásicos com } M E \\
N^{o} \text { de casos/Casos com CaE }\end{array}$} & \multicolumn{2}{|c|}{$\begin{array}{c}\text { Chagásicos sem } M E \\
N^{o} \text { de casos } / \text { Casos com CaE }\end{array}$} & \multicolumn{2}{|c|}{$\begin{array}{c}\text { Não-chagásicos } \\
N^{o} \text { de casos/Casos com CaE }\end{array}$} \\
\hline & 04 & - & 97 & - & 297 & - \\
\hline $25-34$ & 10 & - & 243 & - & 340 & - \\
\hline $35-44$ & 25 & $1(4 \%)$ & 316 & - & 358 & $3(0,08 \%)$ \\
\hline $45-54$ & 21 & - & 264 & $3(1,1 \%)$ & 352 & $6(1,7 \%)$ \\
\hline $55-64$ & 30 & $3(10 \%)$ & 210 & $2(0,9 \%)$ & 284 & $1(0,3 \%)$ \\
\hline $65-74$ & 15 & $1(6,6 \%)$ & 155 & $2(1,2 \%)$ & 249 & $1(0,4 \%)$ \\
\hline 75 em diante & 02 & - & 67 & - & 135 & - \\
\hline Total & 107 & $5(4,6 \%)$ & 1.352 & $7(0,5 \%)$ & 2.015 & $11(0,5 \%)$ \\
\hline
\end{tabular}

\section{DISCUSSÃO}

Conforme os dados das tabelas, a ocorrência de $\mathrm{CaE}$ em nào-chagásicos e em chagásicos sem $\mathrm{ME}$ foi semelhante $(0,5 \%$ para cada grupo). Assim, a simples infecçà pelo Trypanosoma cruzi não deve interferir na frequiência do $\mathrm{CaE}$ em nossa região.
Estes dados concordam com os achados de Chapadeiro e cols ${ }^{4}$, para a associação de tumores em geral (incluindo os esofágicos) e doença de Chagas, também no Triângulo Mineiro. Tal concordància não se deu, todavia, com as observações de Lustig e cols.", na Argentina. Estes autores, através de testes sorológicos, detectaram 49 chagásicos 
entre 474 portadores de tumores que procuraram um Instituto de Oncologia de Buenos Aires; no mesmo período e na mesma instituição, outros 422 indivíduos, sem patologia tumoral, foram atendidos; destes, 25 eram chagásicos. Com base nos referidos dados, afirmam que a freqüência de tumores foi significativamente maior nos chagásicos, em relação aos sorologicamente negativos $(\mathrm{p}<0,05)$. Entre os pacientes com algum tumor, $48 \%$ dos chagásicos tinham vivido por longo tempo no norte da Argentina (zona endêmica da tripanossomose) contra apenas $14 \%$ dos nàochagásicos. Comparando as sedes dos tumores, os mesmos autores ainda observaram freqüência significativamente maior de câncer do esôfago e do colo uterino no grupo chagásico. De acordo com os citados autores, tais diferenças poderiam deverse, em parte, à discrepância geográfica já citada: no norte do país, onde viveu quase metade dos chagásicos, as citadas formas de câncer são mais frequiêntes.

Alguns aspectos dificultam, a nosso ver, a comparaçào entre nossos achados e os de Lustig e cols. 1) a casuística destes autores foi representada por uma amostra relativamente pequena e selecionada de chagásicos; 2) as populações de chagásicos e não-chagásicos analisadas parecem muito heterogêneas geograficamente; 3) os pacientes nào-chagásicos constituíram um grupo pouco representativo de controles, sob o ponto de vista qualitativo; 4) quanto à relação com o $\mathrm{ME}$, este, como outros "megas" chagásicos, é infrequiente na Argentina. De qualquer forma, não se pode, evidentemente, excluir que a infecção chagásica naquele pais se comporte de forma diversa da observada entre nós também no que respeita aos tumores em geral e ao $\mathrm{CaE}$.

Em nosso material, foi altamente significativo o aumento da frequêencia do $\mathrm{CaE}$ associado ao $\mathrm{MECh}$, em relação aos controles e aos chagásicos sem ME. Esta maior frequiência de $\mathrm{CaE}$ em chagásicos com $\mathrm{ME}$ que nos sem $\mathrm{ME}$, nào se explica pela hipótese de maior sobrevida daqueles que destes. De fato, o pareamento por faixa etária (Tabela 2) ainda mostra maior frequiência de $\mathrm{CaE}$ nos chagásicos com "megas" (a não ser no grupo de 45 a 54 anos, em que não tivemos nenhum caso de $\mathrm{CaE}$ dentre os portadores de $\mathrm{ME}$ ). $\mathrm{O}$ mesmo se pode dizer em relação aos não-chagásicos. A propósito; este predomínio de $\mathrm{CaE}$ em portadores de $\mathrm{ME}$, relativamente à populaçào geral, já fora observado ou sugerido por Câmara-Lopes ${ }^{3}$, Rezen- de", e Pinotti e cols. ${ }^{7}$, com base em dados clínicos e cirúrgicos.

\section{SUMMARY}

We analised, in autopsies, the occurrence of esophagic carcinoma in non-chagasic patients and in chagasic ones with and without megaesophagus.

The frequency of esophagic carcinoma was significantly higher in patients with megaesophagus than in controls and chagasic patients without megaesophagus.

The association between carcinoma of the esophagus and chagasic infection per se was not statistically significant.

Key words: Megaesophagus. Carcinoma of esophagus. Chagas disease. Cancer. Autopsies.

\section{REFERENCIAS BIBLIOGRĀFICAS}

1. Andrade $Z$ A. Patologia de la enfermedad de Chagas. In: $12^{\circ}$ Congresso Argentino de Gastroenterologia, Buenos Aires, 2-7 de novembro de 1975. Citado por: Rezende, J.M."

2. Brandalise N A, Leonardi L S, Torre C A D, Morisot $P$. Carcinoma do esôfago em megaesôfago chagásico após operação de Merendino. Revista Paulista de Medicina 83:169-172, 1974.

3. Camara Lopes L H. Carcinoma of the esophagus as a complication of megaesophagus. An analysis of seven cases. American Journal of Digestive Diseases 6:742-756, 1961 .

4. Chapadeiro E, Lopes E R, Mesquita P M, Pereira F E L. Ocorrência de neoplasias malignas associadas à doença de Chagas. O Hospital 66:791-794, 1964.

5. Huggins D. Carcinoma do esôfago associado ao megaesôfago chagásico (relato de um caso). Anais do Instituto de Higiene e Medicina Tropical (Lisboa) 4:1-4, 1976.

6. Lustig E S, Puricelli L, Bal E, Lanseti J C. Association of Chagas disease and cancer. Medicina (Buenos .Aires) 40:43-46, 1980.

7. Pinotti H W, Pollara W M, Gemperli R, Raia A A. O problema do câncer no megaesôfago. Revista da Associação Médica Brasileira 26:379-381, 1980.

8. Raia A A. Megaesôfago. In: Zerbini E J. Clinica Cirúrgica Alípio Corrêa Netto, 3a edição, vol. 4. Sarvier, São Paulo, p. 181-204, 1974.

9. Rezende, J M. Manifestações digestivas da doença de Chagas, In: Dani R, Castro L P. Gastróenterologia Clínica, volume 2. Guanabara Koogan, Rio de Janeiro, p. 1141-1168, 1981. 
Rocha A, Almeida HO, Esper FE, Moraes DM, Santos EP, Teixeira VPA. A associação entre megaesófago e carcinoma de esôfago. Revista da Sociedade Brasileira de Medicina Tropical 16:94-97, Abr-Jun, 1983

10. Rocha A, Henrique D, Borges E G, Oliveira V L, Soares V M G, Moraes A T, Teixeira V P A, Almeida H O. Complicações do megacólon e megaesôfago chagásico observadas em necropsia. Revista Goiana de Medicina 27:53-62, 1981.

11. Wychulis A R, Woolam G L, Andersen H A, Ellis F
H, Jr. Achalasia and carcinoma of the esophagus. JAMA 215:1638-1641, 1971 .

12. Zillioto Jr, A, Kunzle J E, Araujo E J, Ricci G O. Carcinoma espinocelular de esôfago. Experiência em 56 casos. Revista da Associação Médica Brasileira 27:253-256, 1981. 